\title{
Supersymmetric warped conformal field theory
}

\author{
Bin Chen, ${ }^{1,2,3}$ Peng-Xiang Hao, ${ }^{1}$ and Yan-jun Liu $\oplus^{1}$ \\ ${ }^{1}$ Department of Physics and State Key Laboratory of Nuclear Physics and Technology, Peking University, \\ 5 Yiheyuan Rd, Beijing 100871, People's Republic of China \\ ${ }^{2}$ Collaborative Innovation Center of Quantum Matter, 5 Yiheyuan Rd, \\ Beijing 100871, People's Republic of China \\ ${ }^{3}$ Center for High Energy Physics, Peking University, 5 Yiheyuan Rd, \\ Beijing 100871, People's Republic of China
}

(Received 10 June 2020; accepted 9 September 2020; published 23 September 2020)

\begin{abstract}
In this work, we study the supersymmetric warped conformal field theory in two dimensions. We show that the Hofman-Strominger theorem on symmetry enhancement could be generalized to the supersymmetric case. More precisely, we find that within a chiral superspace $\left(x^{+}, \theta\right)$, a two-dimensional field theory with two translational invariance and a chiral scaling symmetry can have enhanced local symmetry, under the assumption that the dilation spectrum is discrete and non-negative. Similar to the pure bosonic case, there are two kinds of minimal models, one being $N=(1,0)$ supersymmetric conformal field theories, while the other being $N=1$ supersymmetric warped conformal field theories (SWCFT). We study the properties of SWCFT, including the representations of the algebra, the space of states, and the correlation functions of the superprimaries.
\end{abstract}

DOI: 10.1103/PhysRevD.102.065016

\section{INTRODUCTION}

Symmetry plays an essential role in quantum field theories. The theories with more symmetries could be better constrained, such that their dynamics might be investigated even nonperturbatively. For example, the supersymmetric field theories have better UV behaviors, and the conformal invariant theories are expected to be solvable in the framework of conformal bootstrap.

In two dimensions (2D), the global symmetries in a quantum field theory with scaling symmetry could be enhanced. As shown by J. Polchinski in the late 1980s [1], a 2D Poincaré invariant QFT with a scale invariance could become conformal invariant, provided that the theory is unitary and the dilation spectrum is discrete and nonnegative. In 2011, D. Hofman and A. Strominger [2] relaxed the requirement of Lorentz invariance and studied the enhanced symmetries of the theory with chiral scaling. They obtained two kinds of minimal theories, one being the two-dimensional conformal field theory $\left(\mathrm{CFT}_{2}\right)$ [3] and the other being the so-called the warped conformal field theory $\left(\mathrm{WCFT}_{2}\right)$ [4]. In a warped $\mathrm{CFT}_{2}$, the global symmetry group is $S L(2, R) \times U(1)$, and it is enhanced

Published by the American Physical Society under the terms of the Creative Commons Attribution 4.0 International license. Further distribution of this work must maintain attribution to the author(s) and the published article's title, journal citation, and DOI. Funded by SCOAP ${ }^{3}$. to an infinite-dimensional group generated by an VirasoroKac-Moody algebra. Very recently, the symmetry enhancement in 2D QFT was generalized to the cases with global translations and anisotropic scaling symmetries [5]. In such 2D Galilean field theories with anisotropic scaling, the enhanced local symmetries are generated by the infinite dimensional spin- $\ell$ Galilean algebra with possible central extensions, under the assumption that the dilation operator is diagonalizable and has a discrete and non-negative spectrum.

$\mathrm{WCFT}_{2}$ has rich structures similar to $\mathrm{CFT}_{2}$. Though they are not Lorentzian invariant, $\mathrm{WCFT}_{2}$ shares the modular covariance like $\mathrm{CFT}_{2}$. For finite temperature $\mathrm{WCFT}_{2}$ defined on a torus, the modular property can be used to evaluate the density of states at high temperature, which gives a Cardy-like formula for the thermal entropy of $\mathrm{WCFT}_{2}$ [4]. Due to the infinite symmetries, $\mathrm{WCFT}_{2}$ is highly constrained. The form of the two- and three-point functions are determined by the global warped conformal symmetry, while the four-point functions can be determined up to an arbitrary function of the cross ratio [6]. Specific models of $\mathrm{WCFT}_{2}$ include chiral Liouville gravity [7], free Weyl fermion [8,9], free scalars [10], and also the Sachdev-Ye-Kitaev models with complex fermions $[11,12]$. For the study of other aspects of $\mathrm{WCFT}_{2}$, see [11,13-18].

On the other hand, $\mathrm{WCFT}_{2}$ plays an important role in the study of holography beyond the usual AdS/CFT correspondence. In [19], it has been shown that under the Compère-Song-Strominger (CSS) boundary conditions, the asymptotic symmetry group of the anti-de Sitter $\left(\mathrm{AdS}_{3}\right)$ 
gravity is generated by an Virasoro-Kac-Moody algebra. This leads to the conjecture that under the CSS boundary conditions, the $\mathrm{AdS}_{3}$ gravity could be dual to a holographic warped conformal field theory. This $\mathrm{AdS}_{3} /$ WCFT correspondence has been studied in [14,16,20-24]. Moreover, $\mathrm{WCFT}_{2}$ could also appear in the $\mathrm{WAdS}_{3} / \mathrm{WCFT}_{2}$ correspondence [25-28], in which the bulk gravity is a threedimensional topological massive gravity.

In this paper, we would like to generalize the study on $\mathrm{WCFT}_{2}$ to the supersymmetric case. We first study the supersymmetries on the warped flat geometry [9] in two dimensions, which is essentially equivalent to a NewtonCartan geometry [29-34] with an additional scaling structure. The supersymmetrization could be done by including Grassmannian coordinates into the bosonic directions to make warped "superspace." However, it turns out that the minimal supersymmetry could be realized in a chiral $N=$ $(1,0)$ superspace. We then study the enhanced local symmetries, following the approach developed in [1,2]. Just as in bosonic case, we find two classes of minimal enhanced algebra. One generates the local symmetries of $N=(1,0) \mathrm{SCFT}_{2}$, while the other one generates the symmetries of the supersymmetric warped conformal field theory $\left(\mathrm{SWCFT}_{2}\right)$. Furthermore, we discuss the radial quantization and the state-operator correspondence in $\mathrm{SWCFT}_{2}$, analogous to the usual $\mathrm{WCFT}_{2}$ case. We study the correlation functions of superprimaries in $\mathrm{SWCFT}_{2}$ as well. We notice that the correlation functions share the similar structure as the ones in the holomorphic sector of $N=(1,0) \mathrm{SCFT}_{2}$, with additional modifications from $U(1)$ symmetry.

The remaining parts are organized as follows. In Sec. II, we discuss the supersymmetries on the warped geometry and set our notations. In Sec. III, we generalize the Hofman-Strominger theorem to the supersymmetric case and show that the global symmetries are enhanced to the local ones. In Sec. IV, we consider the Hilbert space and the representation of the NS sector of the $\mathrm{SWCFT}_{2}$. After establishing the state-operator correspondence, we discuss the transformations of the superprimaries. Then, we calculate the two-point functions and three-point functions of the superprimary operators in the NS sector of the SWCFT and discuss the higher-point functions. We conclude and give some discussions in Sec. V. In the Appendix, we discuss the conserved currents in the superspace and show that we can consistently work in the $N=(1,0)$ superspace.

\section{SUPERSYMMETRIES ON WARPED GEOMETRY}

Let us start from a two-dimensional unitary local field theory with translational invariance and a chiral scaling symmetry. The transformation of coordinates under these symmetries are

$$
x^{a} \rightarrow x^{a}+\delta^{a}, \quad x^{a} \rightarrow \lambda^{a}{ }_{b} x^{b},
$$

where $\lambda_{b}^{a}$ is a scaling matrix,

$$
\lambda_{b}^{a}=\left(\begin{array}{cc}
\lambda & 0 \\
0 & 1
\end{array}\right)
$$

As shown in [2], the theory would have enhanced local symmetries. There are two kinds of minimal theories. One kind is the two-dimensional conformal field theory $\left(\mathrm{CFT}_{2}\right)$, while the other kind is the two-dimensional warped conformal field theory $\left(\mathrm{WCFT}_{2}\right)$. For $\mathrm{WCFT}_{2}$, in addition to the symmetries (2.1), there is a generalized boost symmetry,

$$
x^{a} \rightarrow \Lambda^{a}{ }_{b} x^{b},
$$

where $\Lambda_{b}^{a}$ is the boost matrix,

$$
\lambda^{a}{ }_{b}=\left(\begin{array}{ll}
1 & 0 \\
v & 1
\end{array}\right)
$$

The $\mathrm{WCFT}_{2}$ can be defined consistently in a warped geometry, which is a variant of the Newton-Cartan geometry with an additional scaling structure [4]. In the warped geometry, there are one vector and one 1 form,

$$
q^{a}=\left(\begin{array}{l}
0 \\
1
\end{array}\right), \quad q_{a}=(1,0)
$$

which are invariant under the boost. And there is an antisymmetric tensor $h_{a b}$, which is also invariant under the boost,

$h_{a b}=\left(\begin{array}{cc}0 & 1 \\ -1 & 0\end{array}\right), \quad h^{a b}=-h_{a b=}\left(\begin{array}{cc}0 & -1 \\ 1 & 0\end{array}\right)$.

$h_{a b}$ can be used to lower the indices, but one should keep in mind that $h_{a b}$ is not the metric of the warped geometry.

In the warped geometry, one may define the fermionic representations. The first step is to consider the gamma matrix algebra. The gamma matrix algebra is given by the warped Clifford algebra,

$$
\left\{\Gamma^{a}, \Gamma^{b}\right\}=2 q^{a} q^{b}
$$

where the gamma matrices are ${ }^{1}[8,9]$

$$
\Gamma^{0}=\left(\begin{array}{cc}
0 & 0 \\
1 & 0
\end{array}\right), \quad \Gamma^{1}=\left(\begin{array}{cc}
1 & 0 \\
0 & -1
\end{array}\right) .
$$

\footnotetext{
${ }^{1}$ Actually, there exists a trivial representation in which there is only one supercharge. However, the algebra of the enhanced symmetry in the trivial representation can only satisfy the graded Jacobi identity when the Kac level vanishes. We shall not be considering this case in the present work.
} 
The lower-index gamma matrices are defined by

$$
\Gamma_{a}=h_{a b} \Gamma^{b} .
$$

This definition proves quite useful as it allows us to define the boost generator as

$$
\bar{B}=\frac{1}{8} h_{a b}\left[\Gamma^{a}, \Gamma^{b}\right] .
$$

One can check that it acts on the gamma matrices as they are in a vector representation,

$$
\left[\bar{B}, \Gamma^{c}\right]=q_{a} \Gamma^{a} q^{c}, \quad \text { or } \quad\left[\bar{B}, \Gamma_{c}\right]=-q^{a} \Gamma_{a} q_{c} .
$$

The operators generating the translations will be donated by $H_{a}=\left(H_{0}, H_{1}\right)=(H, \bar{P})$, and they are of course in a vector representation of the boost generator,

$$
\left[\bar{B}, H^{c}\right]=q_{a} H^{a} q^{c}, \quad \text { or } \quad\left[\bar{B}, H_{c}\right]=-q^{a} H_{a} q_{c} .
$$

The two-dimensional spinor space is spanned by $\Psi_{0}, \Psi_{1}$ as follows:

$$
\Psi_{A}=\left(\begin{array}{c}
\Psi_{0} \\
\Psi_{1}
\end{array}\right)
$$

From this, it is easy to see that

$$
\bar{B} \Psi_{0}=0, \quad \bar{B} \Psi_{1}=\frac{1}{2} \Psi_{0}
$$

The definition for the dual representation is

$$
\bar{\Psi}^{A}=\epsilon^{A B} \Psi_{B}=\left(\Psi^{0}, \Psi^{1}\right)=\left(\Psi_{1},-\Psi_{0}\right),
$$

where the $\epsilon^{A B}$ is given by

$$
\epsilon^{A B}=-\epsilon_{A B}=\left(\begin{array}{cc}
0 & 1 \\
-1 & 0
\end{array}\right) .
$$

One can easily show that the quantity $\bar{\Psi} \Psi$ is a scalar under the boost.

Now, let us introduce the supercharge operator $Q=\left(Q_{0}, Q_{1}\right)^{T}$. The commutators of supercharges are

$$
i\left\{Q_{A}, \bar{Q}^{B}\right\}=2\left(\Gamma^{a} H_{a}\right)_{A}{ }^{B} .
$$

They can be written in terms of the component operators,

$i\left\{Q_{1}, Q_{1}\right\}=2 H, \quad i\left\{Q_{0}, Q_{1}\right\}=2 \bar{P}, \quad i\left\{Q_{0}, Q_{0}\right\}=0$.

From these commutators, one can easily find that $H$ and $Q_{1}$ are superpartners under the action of $Q_{1}$, so are $\bar{P}$ and $Q_{0}$.
For simplicity, we will donate $Q_{1}$ by $Q_{+}$and $Q_{0}$ by $Q_{-}$ in the following discussion. Moreover, we will donate $x^{a}=$ $\left(x^{0}, x^{1}\right)$ by $x^{a}=\left(x^{+}, x^{-}\right)$and the Grassmannian coordinates by

$$
\theta^{A}=\left(\theta^{+}, \theta^{-}\right)
$$

A general superfield is defined on the superspace and can be expanded as a power series in $\theta^{+}$and $\theta^{-}$,

$$
\begin{aligned}
\Phi(\mathbf{z}) \equiv & \Phi\left(x^{+}, x^{-}, \theta^{+}, \theta^{-}\right)=A_{1}\left(x^{+}, x^{-}\right)+\theta^{+} A_{2}\left(x^{+}, x^{-}\right) \\
& +\theta^{-} A_{3}\left(x^{+}, x^{-}\right)+\theta^{+} \theta^{-} A_{4}\left(x^{+}, x^{-}\right) .
\end{aligned}
$$

The transformation of any field $\Phi$ under the generator $G$ is given by

$$
\delta_{\epsilon} \Phi=i[\epsilon G, \Phi]
$$

In the Appendix, we discuss the conserved charges of the theory in the superspace and their corresponding supercurrents. We find that there exists a minimal superspace in which the right-moving supersymmetry can be turned off consistently. As we show in the next section, even only with the supersymmetry in the left-moving sector, the rightmoving global symmetry gets enhanced and supersymmetrized as well.

\section{ENHANCED SYMMETRIES}

In this section, we study the enhanced symmetry of two-dimensional quantum field theory, whose global symmetry is generated by the left-moving translation $H$, the dilation $D$, the right-moving translation $\bar{P}$, and the supersymmetries $Q_{+}$. We will work in the chiral superspace $\left(x^{+}, x^{-}, \theta^{+}\right)$. For simplicity, we denote $\theta^{+}=\theta$. Now for the global charges, their related supercurrents depend only on one Grassmannian coordinate $\theta$; we will discuss the enhanced symmetry in this $N=1$ chiral superspace. As in [2], we assume that the eigenvalue spectrum of $D$ is discrete and non-negative, and there exists a complete basis of $N=1$ local superfields $\Phi_{i}$.

A general superfield can be expanded as

$$
\Phi\left(x^{+}, x^{-}, \theta\right)=\varphi\left(x^{+}, x^{-}\right)+\theta \psi\left(x^{+}, x^{-}\right) .
$$

It satisfies

$$
\begin{gathered}
i\left[H, \Phi_{i}\right]=\partial_{+} \Phi_{i}, \quad i\left[\bar{P}, \Phi_{i}\right]=\partial_{-} \Phi_{i} \\
i\left[D, \Phi_{i}\right]=\left(x^{+} \partial_{+}+\frac{\theta}{2} \partial_{\theta}+\lambda_{i}\right) \Phi_{i} \\
i\left[\epsilon Q_{+}, \Phi_{i}\right]=\epsilon\left(\partial_{\theta}-\theta \partial_{+}\right) \Phi_{i} \\
i\left[\epsilon Q_{-}, \Phi_{i}\right]=\epsilon\left(-2 \theta \partial_{-}\right) \Phi_{i}
\end{gathered}
$$


where $\lambda_{i}$ is the superweight of $\Phi_{i}$ and $\int_{C} d \Phi_{i}=0$ for any closed contour $C$. The translational plus the dilational invariance and the supersymmetry restrict the form of the vacuum two-point functions,

$\left\langle\Phi_{i}\left(x^{+}, x^{-}, \theta\right) \Phi_{j}\left(x^{+\prime}, x^{-\prime}, \theta^{\prime}\right)\right\rangle=\frac{f_{i j}\left(x^{-}-x^{-\prime}\right)}{\left(x^{+}-x^{+\prime}-\theta \theta^{\prime}\right)^{\lambda_{i}+\lambda_{j}}}$,

where $f_{i j}$ are some unknown functions.

\section{A. From left global symmetries to local symmetries}

The global charges $H, D, \bar{P}$ are associated to the supercurrents $\mathbb{H}, \mathbb{D}$, and $\overline{\mathbb{P}}$, respectively. All of these supercurrents have shift freedom [2], which can be used to "gauge" the currents to satisfy the canonical commutation relations,

$$
\begin{aligned}
i\left[H, \mathbb{U}_{ \pm}\right] & =\partial_{+} \mathbb{H}_{ \pm}, \quad i\left[H, \overline{\mathbb{P}}_{ \pm}\right]=\partial_{+} \overline{\mathbb{P}}_{ \pm}, \\
i\left[H, \mathbb{D}_{ \pm}\right] & =\partial_{+} \mathbb{D}_{ \pm}-\mathbb{U}_{ \pm} \cdot \\
i\left[\bar{P}, \mathbb{U}_{ \pm}\right] & =\partial_{-} \mathbb{H}_{ \pm}, \quad i\left[\bar{P}, \overline{\mathbb{P}}_{ \pm}\right]=\partial_{-} \overline{\mathbb{P}}_{ \pm}, \\
i\left[\bar{P}, \mathbb{D}_{ \pm}\right] & =\partial_{-} \mathbb{D}_{ \pm} .
\end{aligned}
$$

This implies that $\mathbb{M}_{ \pm}, \overline{\mathbb{P}}_{ \pm}$are local operators, but $\mathbb{D}_{ \pm}$must have an explicit dependence on the $x^{+}$coordinate. The weights of the global charges (A2)-(A3) imply

$$
\begin{aligned}
& i\left[D, \mathbb{M}_{+}\right]=x^{+} \partial_{+} \mathbb{M}_{+}+\frac{\theta}{2} \partial_{\theta} \mathbb{M}_{+}+\frac{3}{2} \mathbb{\boxplus}_{+},
\end{aligned}
$$

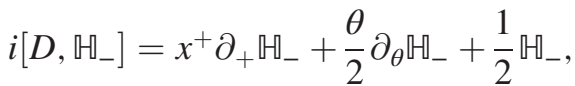

$$
\begin{aligned}
& i\left[D, \overline{\mathbb{P}}_{+}\right]=x^{+} \partial_{+} \overline{\mathbb{P}}_{+}+\frac{\theta}{2} \partial_{\theta} \overline{\mathbb{P}}_{+}+\frac{1}{2} \overline{\mathbb{P}}_{+}, \\
& i\left[D, \overline{\mathbb{P}}_{-}\right]=x^{+} \partial_{+} \overline{\mathbb{P}}_{-}+\frac{\theta}{2} \partial_{\theta} \overline{\mathbb{P}}_{-}-\frac{1}{2} \overline{\mathbb{P}}_{-}, \\
& i\left[D, \mathbb{D}_{+}\right]=x^{+} \partial_{+} \mathbb{D}_{+}+\frac{\theta}{2} \partial_{\theta} \mathbb{D}_{+}+\frac{1}{2} \mathbb{D}_{+}, \\
& i\left[D, \mathbb{D}_{-}\right]=x^{+} \partial_{+} \mathbb{D}_{-}+\frac{\theta}{2} \partial_{\theta} \mathbb{D}_{-}-\frac{1}{2} \mathbb{D}_{-} \text {. }
\end{aligned}
$$

The $\mathbb{D}_{ \pm}$'s have explicit coordinate dependence. Let us write the current in terms of local operators as in [2]. Defining $\mathbb{S}_{ \pm}$by

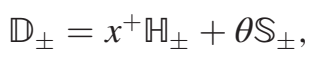

one can easily find that

$$
i\left[H, \mathbb{S}_{ \pm}\right]=\partial_{+} \mathbb{S}_{ \pm}, \quad i\left[\bar{P}, \mathbb{S}_{ \pm}\right]=\partial_{-} \mathbb{S}_{ \pm},
$$

and
$i\left[D, \mathbb{S}_{+}\right]=x^{+} \partial_{+} \mathbb{S}_{+}+\mathbb{S}_{+}, \quad i\left[D, \mathbb{S}_{-}\right]=x^{+} \partial_{+} \mathbb{S}_{-}$.

So we conclude $\left(\mathbb{S}_{+}, \mathbb{S}_{-}\right)$are local operators of a weight $(1,0)$.

The conservations of the dilation current and left-translation current yield

$$
\begin{aligned}
& \partial_{+} \mathbb{D}_{-}+\partial_{-} \mathbb{D}_{+}=x^{+}\left(\partial_{+} \mathbb{W}_{-}+\partial_{-} \mathbb{W}_{+}\right)+\theta\left(\partial_{+} \mathbb{S}_{-}\right. \\
& \left.+\partial_{-} \mathbb{S}_{+}\right)+\mathbb{H}_{-} \\
& =0 \text {, }
\end{aligned}
$$

which leads to

$$
\mathbb{W}_{-}=-\theta\left(\partial_{-} \mathbb{S}_{+}+\partial_{+} \mathbb{S}_{-}\right) .
$$

Then we use the shift freedom in the currents to shift away $\mathbb{S}_{+}$,

$\mathbb{H}_{ \pm} \rightarrow \mathbb{U}_{ \pm} \pm \theta \partial_{ \pm} \mathbb{S}_{+}, \quad \mathbb{D}_{ \pm} \rightarrow \mathbb{D}_{ \pm} \pm \theta \partial_{ \pm}\left(x^{+} \mathbb{S}_{+}\right)$.

One can check that the commutators and the conservations of the currents remain consistent. Now, the Eq. (3.10) becomes

$$
\mathbb{D}_{+}=x^{+} \mathbb{W}_{+}, \quad \mathbb{D}_{-}=x^{+} \mathbb{Q}_{-}+\theta \mathbb{S}_{-},
$$

and

$$
\mathbb{H}_{-}=-\theta \partial_{+} \mathbb{S}_{-} .
$$

Because $\mathbb{S}_{-}$is a local operator of a weight zero, then from the general form of the two-point function, we have

$$
\left\langle\mathbb{S}_{-} \mathbb{S}_{-}\right\rangle=f_{\mathbb{S}_{-}}\left(x^{-}\right),
$$

which implies

$$
\mathbb{U}_{-}=0 .
$$

Assuming

$$
\mathbb{H}_{+}\left(x^{+}, x^{-}, \theta\right)=h_{0}\left(x^{+}, x^{-}\right)+2 \theta h_{1}\left(x^{+}, x^{-}\right),
$$

the conservation law and the Eq. (3.19) yield

$$
\mathbb{W}_{+}\left(x^{+}, x^{-}, \theta\right)=h_{0}\left(x^{+}\right)+2 \theta h_{1}\left(x^{+}\right) .
$$

This fact immediately leads to the existence of two sets of conserved charges. Defining 


$$
T_{\xi}=-\frac{1}{2 \pi} \int d x^{+} d \theta \xi\left(x^{+}, \theta\right) \mathbb{H}_{+},
$$

where $\xi=\frac{1}{2} \alpha\left(x^{+}\right)+\theta a\left(x^{+}\right)$with $\alpha$ and $a$ being the function of $x^{+}$, we have

$$
\begin{aligned}
& T_{0 a}=-\frac{1}{2 \pi} \int d x^{+} a\left(x^{+}\right) h_{0}, \\
& T_{1 \alpha}=-\frac{1}{2 \pi} \int d x^{+} \alpha\left(x^{+}\right) h_{1} .
\end{aligned}
$$

There is another set of conserved charges,

$$
\bar{J}_{\chi}=\frac{1}{2 \pi} \int d x^{-} \chi\left(x^{-}\right) \mathbb{S}_{-},
$$

which may lead to other symmetries [2,9]. As we are going to discuss the minimal algebra, we choose $\mathbb{S}_{-}=0$.

The algebra spanned by the conserved bosonic charges $T_{1 \alpha}$ has been done in [2],

$$
i\left[T_{1 \alpha}, T_{1 \beta}\right]=T_{1\left(\alpha^{\prime} \beta-\alpha \beta^{\prime}\right)}+\frac{c_{0}}{48 \pi} \int d x^{+}\left(\alpha^{\prime \prime} \beta^{\prime}-\alpha^{\prime} \beta^{\prime \prime}\right),
$$

where the prime denotes the derivative with respect to $x^{+}$, $\alpha^{\prime} \equiv \partial_{+} \alpha$. This is the same as the algebra of the left-moving conformal generators on the Minkowski plane with the central charge $c_{0}$.

Let us now work out the algebra spanned by adding the fermionic charges $T_{0 a}$. The global charges are

$$
\begin{gathered}
H=-\frac{1}{4 \pi} \int d x^{+} d \theta 1 \cdot \mathbb{W}_{+}=-\frac{1}{2 \pi} \int d x^{+} h_{1}, \\
D=-\frac{1}{4 \pi} \int d x^{+} d \theta \theta x^{+} \cdot \mathbb{H}_{+}=-\frac{1}{2 \pi} \int d x^{+} x^{+} h_{1}, \\
Q_{+}=-\frac{1}{2 \pi} \int d x^{+} d \theta \theta \mathbb{}_{+}=-\frac{1}{2 \pi} \int d x^{+} h_{0} .
\end{gathered}
$$

The actions of $H$ and $D$ on $\mathbb{H}_{+}$imply

$$
\begin{gathered}
i\left[H, T_{0 a}\right]=-T_{0 a^{\prime}}, \\
i\left[D, T_{0 a}\right]=-T_{0\left(\frac{a}{2}-a^{\prime} x^{+}\right)} .
\end{gathered}
$$

This in turn implies that the action of $T_{0 a}$ on $h_{1}$ is

$$
i\left[h_{1}, T_{0 a}\right]=-\frac{3}{2} a^{\prime} h_{0}-\frac{1}{2} a \partial_{+} h_{0}+\partial_{+}^{2} O_{a} .
$$

Furthermore, we have

$$
i\left[T_{1 \alpha}, T_{0 a}\right]=T_{0\left(\frac{\alpha^{\prime} a}{2}-\alpha a^{\prime}\right)}-\frac{1}{2 \pi} \int d x^{+} \alpha \partial_{+}^{2} O_{a} .
$$

The scaling symmetry plus the locality imply that $O_{a}$ must be of the form $O_{a}=c_{1} a$ with $c_{1}$ being a local operator of weight $\frac{1}{2}$. But the Jacobi identity with the third operator $T_{1 \beta}$ implies that $c_{1}=0$. So we arrive at

$$
i\left[T_{1 \alpha}, T_{0 a}\right]=T_{0\left(\frac{\alpha^{\prime} a}{2}-\alpha a^{\prime}\right)} .
$$

Next, the action of $Q_{+}=T_{01}$ on $h_{0}$ is $i\left\{T_{01}, h_{0}\right\}=2 h_{1}$. This implies $i\left\{T_{01}, T_{0 a}\right\}=2 T_{1 a}$ and hence,

$$
i\left\{T_{0 a}, h_{0}\right\}=2 a h_{1}+\partial_{+} g_{a}
$$

where $g_{a}$ is to be determined. Integrating both sides with $-\frac{1}{2 \pi} d x^{+} b\left(x^{+}\right)$gives

$$
i\left\{T_{0 a}, T_{0 b}\right\}=2 T_{1 a b}-\frac{1}{2 \pi} \int d x^{+} b \partial_{+} g_{a} .
$$

The scaling symmetry and the exchange symmetry under $a \leftrightarrow b$ imply $g_{a}=c_{2} a^{\prime}$, where $c_{2}$ is a constant number. The Jacobi identity with the third operator $T_{1 \alpha}$ implies that $c_{2}=\frac{c_{0}}{3}$. Then,

$$
i\left\{T_{0 a}, T_{0 b}\right\}=2 T_{1 a b}+\frac{c_{0}}{6 \pi} \int d x^{+} a^{\prime} b^{\prime} .
$$

We recognize the equations (3.26), (3.34), (3.37) as the superconformal algebra on the Minkowski plane with the central charge $c_{0}$.

\section{B. From right global symmetries to local symmetries}

In general, $\overline{\mathbb{P}}_{ \pm}$can be written in form of

$$
\begin{aligned}
& \overline{\mathbb{P}}_{+}=p_{0}\left(x^{+}, x^{-}\right)+2 \theta p_{1}\left(x^{+}, x^{-}\right), \\
& \overline{\mathbb{P}}_{-}=p_{3}\left(x^{+}, x^{-}\right)+2 \theta p_{2}\left(x^{+}, x^{-}\right) .
\end{aligned}
$$

The fact that $\overline{\mathbb{P}}_{-}$is a local superfield of weight $-\frac{1}{2}$ implies that $p_{2}$ is a weight-zero local field. From the two-point function of $p_{2}$, we get $\partial_{+} p_{2}=0$. The current conservation then implies $\partial_{-} p_{1}=0$. It follows that

$$
p_{1}=p_{1}\left(x^{+}\right), \quad p_{2}=p_{2}\left(x^{-}\right) .
$$

The supersymmetry requires $p_{0}=p_{0}\left(x^{+}\right)$and $p_{3}=0$; hence, $p_{-}$is a singlet under the supersymmetry. Now, we have

$$
\begin{gathered}
\overline{\mathbb{P}}_{+}=p_{0}\left(x^{+}\right)+2 \theta p_{1}\left(x^{+}\right), \\
\overline{\mathbb{P}}_{-}=p_{2}\left(x^{-}\right) .
\end{gathered}
$$


In the case $\overline{\mathbb{P}}_{+}=0$, we have infinitely many charges given by

$$
\bar{T}_{1 \alpha}=\frac{1}{2 \pi} \int d x^{-} \alpha\left(x^{-}\right) p_{2} .
$$

The algebra spanned by $\bar{T}_{1 \alpha}$ gives the right-moving Virasoro algebra on Minkowski plane [2]. In this case, the enhanced local symmetry is generated by the leftmoving super-Virasoro algebra and the right-moving Virasoro algebra. It gives the local symmetry of $N=$ $(1,0) \mathrm{SCFT}_{2}$.

In the case $\overline{\mathbb{P}}_{-}=0$, we have infinitely many left-moving charges,

$$
J_{\eta}=-\frac{1}{2 \pi} \int d x^{+} d \theta \eta\left(x^{+}, \theta\right) \overline{\mathbb{P}}_{+},
$$

where $\eta=\frac{1}{2} \eta\left(x^{+}\right)+\theta c\left(x^{+}\right)$. Then,

$$
\begin{aligned}
& J_{0 c}=-\frac{1}{2 \pi} \int d x^{+} d \theta \theta c \overline{\mathbb{P}}_{+}=-\int d x^{+} c p_{0}, \\
& J_{1 \eta}=-\frac{1}{2 \pi} \int d x^{+} d \theta \cdot \eta \overline{\mathbb{P}}_{+}=-\int d x^{+} \eta p_{1} .
\end{aligned}
$$

The bosonic sector of the algebra are simply [2]

$$
\begin{gathered}
i\left[J_{1 \eta}, J_{1 \chi}\right]=-\frac{k}{8 \pi} \int d x^{+}\left(\chi^{\prime} \eta-\chi \eta^{\prime}\right), \\
i\left[T_{1 \alpha}, J_{1 \eta}\right]=-J_{1 \alpha \eta^{\prime}} .
\end{gathered}
$$

The Eq. (3.47) is a $U(1)$ Kac-Moody current algebra, and the constant $k$ parametrizes the central element.

To find the fermionic sector of the enhanced symmetry, we need to consider other commutators. Firstly, we study the commutator $\left[T_{0 a}, J_{1 \eta}\right]$. Note that the action $Q_{+}=T_{01}$ on $\overline{\mathbb{P}}_{+}$implies

$$
i\left[T_{01}, J_{1 \eta}\right]=\frac{1}{2} J_{0 \eta^{\prime}} .
$$

This in turn implies that the action of $J_{1 \eta}$ on $h_{0}$ is

$$
\left[h_{0}, J_{1 \eta}\right]=\frac{1}{2} \eta^{\prime} p_{0}+\partial_{+} O_{0 \eta} .
$$

The scaling symmetry plus the locality imply that $O_{0 \eta}$ must be of the form $O_{0 \eta}=c_{3} \eta$, where $c_{3}$ is a local operator of weight $\frac{1}{2}$. Consider the zero mode of $J_{1 \eta}(\eta=1) \equiv J_{11}$, which act as $\partial_{-}$, we have $i\left[J_{11}, h_{0}\right]=0=\partial_{+} c_{3}$. This leads to the fact that $c_{3}$ must be independent of $x^{+}$. On the other hand, $c_{3}$ is an operator of weight $\frac{1}{2}$ under the chiral scaling, we conclude that $c_{3}$ must be zero. Now integrating both sides of the Eq. (3.50) with $-\frac{1}{2 \pi} d x^{+} a$ gives

$$
i\left[T_{0 a}, J_{1 \eta}\right]=\frac{1}{2} J_{0\left(a \eta^{\prime}\right)} .
$$

Let us now work out the algebra spanned by $J_{0 c}$. Due to the fact that the zero mode $J_{11}$ acts as $\partial_{-}$, we have $i\left[J_{11}, p_{0}\right]=0$. This implies $i\left[J_{11}, J_{0 c}\right]=0$ and hence,

$$
i\left[J_{0 c}, p_{1}\right]=\partial_{+} X_{c} .
$$

Again, the scaling symmetry plus the locality imply $X_{c}=0$, then

$$
i\left[J_{0 c}, J_{1 \eta}\right]=0 .
$$

We also need the commutator $\left[T_{1 \alpha}, J_{0 c}\right]$. The action of $H$ on $p_{0}$ implies $i\left[H, J_{01}\right]=0$, which in turn implies $i\left[h_{1}, J_{01}\right]=\partial_{+}\left(p_{0}+Y\right)$ with $Y$ a local operator of weight $\frac{1}{2}$. Integrating both sides with $-\frac{1}{2 \pi} d x^{+} \alpha$ gives $i\left[T_{1 \alpha}, J_{01}\right]=$ $\frac{1}{2 \pi} \int d x^{+} \alpha^{\prime}\left(p_{0}+Y\right)$. This gives

$$
i\left[T_{1 \alpha}, p_{0}\right]=-\alpha^{\prime}\left(p_{0}+Y\right)+\partial_{+} Z_{\alpha} .
$$

The scaling symmetry plus the locality implies $Z_{\alpha}$ must be of the form $Z_{\alpha}=c_{4} \alpha$ with $c_{4}$ being a local operator of weight $\frac{1}{2}$. The action of $D_{1}$ on $p_{0}$ implies that $Y=-\frac{p_{0}}{2}$ and $c_{4}=p_{0}$, hence,

$$
i\left[T_{1 \alpha}, p_{0}\right]=\alpha p_{0}^{\prime}+\frac{\alpha^{\prime} p_{0}}{2} .
$$

Then, we have

$$
i\left[T_{1 \alpha}, J_{0 c}\right]=-J_{0\left(\alpha c^{\prime}+\frac{\alpha^{\prime} c}{2}\right)} .
$$

Next we turn to the anticommutator $\left\{T_{0 a}, J_{0 c}\right\}$. The fact $i\left\{Q_{+}, p_{0}\right\}=2 p_{1}$ implies $i\left\{Q_{+}, J_{0 c}\right\}=2 J_{1 c}$. This in turn implies

$$
i\left\{h_{0}, J_{0 c}\right\}=2 c p_{1}+\partial_{+} O_{c} .
$$

The scaling symmetry plus the locality imply $O_{c}$ must be of form $O_{c}=c_{5} c$ with $c_{5}$ being a local operator of weight zero. After integrating both sides with $-\frac{1}{2 \pi} d x^{+} a\left(x^{+}\right)$, we get

$$
i\left\{T_{0 a}, J_{0 c}\right\}=2 J_{1(a c)}-\frac{c_{5}}{2 \pi} \int d x^{+} a c^{\prime} .
$$

The Jacobi identity with the third operator $T_{1 \alpha}$ implies $c_{5}=0$. Finally, we have

$$
i\left\{T_{0 a}, J_{0 c}\right\}=2 J_{1(a c)} .
$$

Finally, we consider the anticommutator $\left\{J_{0 c}, J_{0 d}\right\}$. The scaling symmetry implies that $\left\{J_{01}, p_{0}\right\}=c_{6}$, where $c_{6}$ 
must be a weight-zero constant number. This implies $\left\{J_{01}, J_{0 c}\right\}=-c_{6} \int d x^{+} c$, which in turn gives

$$
i\left\{p_{0}, J_{0 c}\right\}=c_{6} c+\partial_{+} W_{c} .
$$

Again, the scaling symmetry plus the locality imply that $W_{c}=0$. At last, we get

$$
i\left\{J_{0 c}, J_{0 d}\right\}=-c_{6} \int d x^{+} c d .
$$

The appropriate normalizations of $J_{0 c}, J_{1 \eta}$ can always help us to set $c_{6}=-\frac{k}{4 \pi}$, then we recognize (3.47), (3.53), (3.61) as the $U(1)$ super-Kac-Moody algebra (SKMA) on the Minkowski plane with the central charge $k$.

\section{Mode expansion}

The supersymmetric Virasoro-Kac-Moody algebra consists of a super-Virasoro algebra,

$$
\begin{gathered}
i\left[T_{1 \alpha}, T_{1 \beta}\right]=T_{1\left(\alpha^{\prime} \beta-\alpha \beta^{\prime}\right)}+\frac{c_{0}}{48 \pi} \int d x^{+}\left(\alpha^{\prime \prime} \beta^{\prime}-\alpha^{\prime} \beta^{\prime \prime}\right), \\
i\left[T_{1 \alpha}, T_{0 a}\right]=T_{0\left(\frac{\alpha^{\prime} a}{2}-\alpha a^{\prime}\right)}, \\
i\left\{T_{0 a}, T_{0 b}\right\}=2 T_{1 a b}+\frac{c_{0}}{6 \pi} \int d x^{+} a^{\prime} b^{\prime},
\end{gathered}
$$

a super-Kac-Moody algebra,

$$
\begin{gathered}
i\left[J_{1 \eta}, J_{1 \chi}\right]=-\frac{k}{8 \pi} \int d x^{+}\left(\chi^{\prime} \eta-\chi \eta^{\prime}\right), \\
i\left[J_{0 c}, J_{1 \eta}\right]=0, \\
i\left\{J_{0 c}, J_{0 d}\right\}=\frac{k}{4 \pi} \int d x^{+} c d,
\end{gathered}
$$

and the semidirect product of the super-Virasoro and superKac-Moody algebras,

$$
\begin{gathered}
i\left[T_{1 \alpha}, J_{1 \eta}\right]=-J_{1 \alpha \eta^{\prime}}, \\
i\left[T_{0 a}, J_{1 \eta}\right]=\frac{1}{2} J_{0\left(a \eta^{\prime}\right)}, \\
i\left[T_{1 \alpha}, J_{0 c}\right]=-J_{0\left(\alpha c^{\prime}+\frac{\alpha^{\prime} c}{2}\right)}, \\
i\left\{T_{0 a}, J_{0 c}\right\}=2 J_{1(a c)} .
\end{gathered}
$$

Let us put the theory on a cylinder and find the mode expansion of the above algebra. The coordinate transformation is

$$
x^{+}=e^{i \phi} .
$$

Using the new coordinate $\phi$, we choose test functions $\alpha_{n}=\left(x^{+}\right)^{n+1}=e^{i(n+1) \phi}, \quad a_{r}=e^{i\left(r+\frac{1}{2}\right) \phi}, \quad \eta_{n}=e^{i n \phi}$, and $c_{r}=e^{i\left(r-\frac{1}{2}\right) \phi}$, where $n \in \mathbb{Z}$ and $r \in \mathbb{Z}+\frac{1}{2}$ for the NeveuSchwarz (NS) sector or $r \in \mathbb{Z}$ for the Ramond (R) sector. Letting $L_{n}=i T_{1 \alpha_{n}}, G_{r}=i T_{0 a_{r}}, P_{n}=J_{1 \eta_{n}}$, and $S_{r}=J_{0 c_{r}}$, then the commutation relations in terms of the charges $\left\{L_{n}, P_{m}, G_{r}, S_{s}\right\}$ are as follows. ${ }^{2}$ The super-Virasoro algebra is generated by

$$
\begin{gathered}
{\left[L_{m}, L_{n}\right]=(m-n) L_{m+n}+\frac{c}{12} m\left(m^{2}-1\right) \delta_{m+n},} \\
{\left[L_{m}, G_{r}\right]=\left(\frac{m}{2}-r\right) G_{m+r},} \\
\left\{G_{r}, G_{s}\right\}=2 L_{r+s}+\frac{c}{3}\left(r^{2}-\frac{1}{4}\right) \delta_{r+s} .
\end{gathered}
$$

The super-Kac-Moody algebra is generated by

$$
\left[P_{m}, P_{n}\right]=\frac{k}{2} m \delta_{m+n}, \quad\left[P_{m}, S_{r}\right]=0, \quad\left\{S_{r}, S_{s}\right\}=\frac{k}{2} \delta_{r+s} .
$$

The semidirect product part of the super-Virasoro and super-Kac-Moody algebras is generated by $^{3}$

$$
\begin{aligned}
& {\left[L_{m}, P_{n}\right]=-n P_{m+n}, \quad\left[G_{r}, P_{m}\right]=-\frac{m}{2} S_{m+r},} \\
& {\left[L_{m}, S_{r}\right]=-\left(\frac{m}{2}+r\right) S_{m+r}, \quad\left\{G_{r}, S_{s}\right\}=2 P_{r+s} .}
\end{aligned}
$$

These algebras are the same as those appearing in the supersymmetric Wess-Zumino-Witten (SWZW) model [35-38]. We would like to stress that the super-KacMoody algebra in the SWZW model is generated by internal symmetries, while here it is generated by the symmetric transformations in the superspace in $\mathrm{SWCFT}_{2}$. The algebra of the SWZW model consists of two copies (the left moving and the right moving) of algebras, while there is only one copy (left moving) of the algebra in $\mathrm{SWCFT}_{2}$.

Another remarkable point is on the supersymmetry in the right-moving sector. Our construction starts from the leftmoving superspace, but the right-moving sector gets supersymmetrized as well. This could be understood from the diffeomorphism of $\mathrm{WCFT}_{2}$. Recall that the diffeomorphism of $\mathrm{WCFT}_{2}$ is generated by

\footnotetext{
${ }^{2}$ We have set $c_{0}=c$ and $\delta_{m+n}=\delta_{m+n, 0}$.

${ }^{3}$ We have substituted $-P$ for $P$.
} 


$$
x^{+} \rightarrow f\left(x^{+}\right), \quad x^{-} \rightarrow x^{-},
$$

and

$$
x^{+} \rightarrow x^{+}, \quad x^{-} \rightarrow x^{-}+g\left(x^{+}\right) .
$$

The diffeomorphism could be generalize to chiral superspace, such that the supersymmetry in the left-moving sector is transferred to the right-moving sector. Actually, the superconformal transformation in the left-moving sector is [39]

$$
\begin{gathered}
x^{+\prime}=f\left(x^{+}\right)+\theta F\left(x^{+}\right), \\
\theta^{\prime}=\phi\left(x^{+}\right)+\theta p\left(x^{+}\right) .
\end{gathered}
$$

Here, $f\left(x^{+}\right), p\left(x^{+}\right)$are holomorphic functions and $F\left(x^{+}\right)$, $\phi\left(x^{+}\right)$are anticommuting holomorphic functions, satisfying the following relations:

$$
\begin{aligned}
F\left(x^{+}\right) & =\phi\left(x^{+}\right) p\left(x^{+}\right), \\
p\left(x^{+}\right)^{2} & =\partial_{+} f\left(x^{+}\right)+\phi\left(x^{+}\right) \partial_{+} \phi\left(x^{+}\right) .
\end{aligned}
$$

The transformation in the right-moving sector is

$$
x^{-\prime}=x^{-}+g\left(x^{+}\right)+\theta G\left(x^{+}\right),
$$

where $G\left(x^{+}\right)$is an anticommuting holomorphic function. Considering the infinitesimal version of the above transformations, we find that the generators of the superVirasoro-Kac-Moody algebra could be realized by

$$
\begin{aligned}
L_{n} & =\left(x^{+}\right)^{n+1} \partial_{+}+\frac{1}{2}(n+1)\left(x^{+}\right)^{n} \theta \partial_{\theta}, \\
P_{n} & =\left(x^{+}\right)^{n} \partial_{-}, \\
G_{r} & =\left(x^{+}\right)^{r+1 / 2}\left(\partial_{\theta}-\theta \partial_{+}\right), \\
S_{s} & =-2\left(x^{+}\right)^{s-1 / 2} \theta \partial_{-} .
\end{aligned}
$$

They satisfy the above commutation relations without central extensions. Then from the Jacobi identity, the central extensions could be recovered. This fact shows that the chiral superspace $\left(x^{+}, \theta\right)$ is enough for our study.

\section{PROPERTIES OF SWCFT 2}

Now we have found two kinds of minimal theories in $N=(1,0)$ superspace, starting from a 2D QFT with chiral scaling and translation symmetry. One is the $N=(1,0)$ supersymmetric conformal field theory, whose local symmetries consist of a left-moving super-Virasoro algebra (SVA) and a right-moving Virasoro algebra. The other is the supersymmetric warped conformal field theory, whose local symmetries are generated by supersymmetric Virasoro-Kac-Moody algebra (SVCMA). In this section, we discuss the representations of this algebra, the stateoperator correspondence and then the correlation functions in $\mathrm{SWCFT}_{2}$.

\section{A. Primary states and descendants}

In all our subsequent discussions, we consider the NS sector of $\mathrm{SWCFT}_{2}$ and hence, $r, s \in \mathbb{Z}+\frac{1}{2}$. We want to define the states in this theory at $t=0$ by doing radial quantization. For this purpose, we consider the following complex coordinates:

$x^{+}=e^{-i(t-\phi)}=e^{i \phi+t_{E}}, \quad x^{-}=t+2 \gamma(\phi-t)$,

where $t$ is interpreted as the Lorentzian time, and $t_{E}=-i t$ as the Euclidean time. Having an initial state at very early Euclidean time corresponds to insert an operator at $x^{+}=0$. Using translational symmetry, we can further put the operator at $x^{-}=0$. A primary operator $\Phi$ of weight $\Delta$ and charge $Q$ at $x^{+}=0$ corresponds to a state,

$$
\Phi(0,0,0) \sim|\Delta, Q\rangle
$$

In particular, because of the global subalgebra of SVKMA is $\operatorname{osp}(1 \mid 2) \times u(1)$, the identity operator corresponds to the $\operatorname{OSP}(1 \mid 2) \times U(1)$ invariant vacuum. The vacuum state $|0\rangle$ is defined as

$$
\begin{aligned}
L_{n}|0\rangle=0, & n \geq-1, \\
P_{m}|0\rangle=0, & m \geq 0, \\
G_{r}|0\rangle=0, & r \geq-\frac{1}{2}, \\
S_{s}|0\rangle=0, & s \geq \frac{1}{2} .
\end{aligned}
$$

We now construct the representations by considering the states having definite scaling dimensions and $U(1)$ charges. The state $|\Delta, Q\rangle$ is of a scaling dimension $\Delta$ and a charge $Q$,

$$
\begin{aligned}
& L_{0}|\Delta, Q\rangle=\Delta|\Delta, Q\rangle, \\
& P_{0}|\Delta, Q\rangle=Q|\Delta, Q\rangle .
\end{aligned}
$$

Using the algebra obtained previously, we have

$$
\begin{aligned}
L_{0} L_{n}|\Delta, Q\rangle & =(\Delta-n) L_{n}|\Delta, Q\rangle, \\
L_{0} P_{m}|\Delta, Q\rangle & =(\Delta-m) P_{m}|\Delta, Q\rangle, \\
L_{0} G_{r}|\Delta, Q\rangle & =(\Delta-r) G_{r}|\Delta, Q\rangle, \\
L_{0} S_{s}|\Delta, Q\rangle & =(\Delta-s) S_{s}|\Delta, Q\rangle .
\end{aligned}
$$

We can see that the positive modes $L_{n}, P_{m}, G_{r}, S_{s}$ lower the value of the scaling dimension, while the negative modes $L_{-n}, P_{-m}, G_{-r}, S_{-s}$ raise the value of the scaling dimension. 
The superprimary states in the theory are defined to have the following properties:

$$
\begin{aligned}
& L_{n}|\Delta, Q\rangle=0, \\
& P_{n}|\Delta, Q\rangle=0, \quad n>0, \\
& G_{r}|\Delta, Q\rangle=0, \quad r>0, \\
& S_{s}|\Delta, Q\rangle=0, \quad s>0 .
\end{aligned}
$$

The modules (analogue to the Verma modules in $\mathrm{CFT}_{2}$ ) in $\mathrm{SWCFT}_{2}$ are then defined by acting the raising operators $L_{-n}, P_{-m}, G_{-r}, S_{-s}, n, m, r, s>0$ on the primary states. The descendant states at level $N$ are

$$
\begin{aligned}
|\Delta, Q,\{N\}\rangle= & L_{-n_{1}} \cdots L_{-n_{k}} P_{-m_{1}} \cdots P_{-m_{l}} G_{-r_{1}} \\
& \cdots G_{-r_{i}} S_{-s_{1}} \cdots S_{-s_{j}}|\Delta, Q\rangle
\end{aligned}
$$

where $\{N\}$ denotes four sets of $\{n\},\{m\},\{r\}$, and $\{s\}$ and the total level $N$ is the sum of all elements in the sets. A primary module consists of a primary state and all its descendant states.

In our chiral superspace, the superfield has two component fields, which are related to each other by supersymmetric transformation. The states corresponding to the component fields can be obtained from the highest weight state,

$$
\begin{aligned}
& |\varphi\rangle=|\Delta, Q\rangle, \\
& |\psi\rangle=G_{-\frac{1}{2}}|\Delta, Q\rangle .
\end{aligned}
$$

They share the same $P_{0}$ charge,

$$
\mathcal{M}_{\frac{3}{2}}=\left[\begin{array}{cc}
2 \Delta(2 \Delta+1) & 4 Q \\
4 Q & 2 \Delta+\frac{2}{3} c \\
(2 \Delta+1) 2 Q & 4 Q \\
2 \Delta Q & Q \\
2 Q & 2 Q \\
2 Q^{2} & \frac{k}{4}
\end{array}\right.
$$

$$
\begin{aligned}
& P_{0}|\varphi\rangle=Q|\varphi\rangle, \\
& P_{0}|\psi\rangle=Q|\psi\rangle .
\end{aligned}
$$

The matrix of inner products of the states including the descendants defines the SWCFT analogue of the Kac matrix in CFT. We will denote it by $\mathcal{M}_{N}$, and its matrix elements are

$$
\mathcal{M}_{\{N\},\left\{N^{\prime}\right\}}=\left\langle\Delta, Q,\{N\} \mid \Delta, Q,\left\{N^{\prime}\right\}\right\rangle .
$$

At level $\frac{1}{2}$, we have

$\mathcal{M}_{\frac{1}{2}}=\left[\begin{array}{c}\langle\Delta, Q,| G_{\frac{1}{2}} \\ \langle\Delta, Q,| S_{\frac{1}{2}}\end{array}\right]\left[G_{-\frac{1}{2}}|\Delta, Q\rangle, S_{-\frac{1}{2}}|\Delta, Q\rangle\right]=\left[\begin{array}{cc}2 \Delta & 2 Q \\ 2 Q & \frac{k}{2}\end{array}\right]$.

At level 1, we have

$$
\begin{aligned}
\mathcal{M}_{1}= & {\left[\begin{array}{c}
\langle\Delta, Q,| L_{1} \\
\langle\Delta, Q,| S_{\frac{1}{2}} G_{\frac{1}{2}} \\
\langle\Delta, Q,| P_{1}
\end{array}\right] } \\
& \times\left[L_{-1}|\Delta, Q\rangle, G_{-\frac{1}{2}} S_{-\frac{1}{2}}|\Delta, Q\rangle, P_{-1}|\Delta, Q\rangle\right] \\
= & {\left[\begin{array}{ccc}
2 \Delta & 2 Q & Q \\
2 Q & \frac{(2 \Delta+1) k-8 Q^{2}}{2} & \frac{k}{4} \\
Q & \frac{k}{4} & \frac{k}{2}
\end{array}\right] . }
\end{aligned}
$$

At level $\frac{3}{2}$, we have

$\left.\begin{array}{cccc}(2 \Delta+1) 2 Q & 2 \Delta Q & 2 Q & 2 Q^{2} \\ 4 Q & Q & 2 Q & \frac{k}{4} \\ \frac{(2 \Delta+1) k}{2} & 2 Q^{2} & \frac{k}{2} & \frac{k Q}{2} \\ 2 Q^{2} & k \Delta & 0 & k \Delta \\ \frac{k}{2} & 0 & \frac{k}{2} & 0 \\ \frac{k Q}{2} & k \Delta & 0 & \frac{k^{2}}{4}\end{array}\right]$,

which is in the base,

$$
\left\{L_{-1} G_{-\frac{1}{2}}|\Delta, Q\rangle, G_{-\frac{3}{2}}|\Delta, Q\rangle, L_{-1} S_{-\frac{1}{2}}|\Delta, Q\rangle, P_{-1} G_{-\frac{1}{2}}|\Delta, Q\rangle, S_{-\frac{3}{2}}|\Delta, Q\rangle, P_{-1} S_{-\frac{1}{2}}|\Delta, Q\rangle\right\} .
$$

We can derive some simple unitarity bounds on the plane charges by requiring the norm of the states to be nonnegative. First, we have

$$
\begin{gathered}
\left\|L_{n} \mid \Delta, Q>\right\| \geq 0 \Rightarrow \Delta \geq 0, \quad c \geq 0, \\
\left\|P_{n} \mid \Delta, Q>\right\| \geq 0 \Rightarrow Q \in \mathbb{R}, \quad k \geq 0 .
\end{gathered}
$$

Furthermore, the matrix $\mathcal{M}_{\frac{1}{2}}$ gives

$$
k \Delta-4 Q^{2} \geq 0 \Rightarrow k \geq \frac{4 Q^{2}}{\Delta} .
$$

Recently, it was found that in order to have a bulk dual, the holographic warped CFT must have a negative 
Kac-Moody level. This seems to indicate a nonunitarity. The interesting point is that such nonunitarity is controllable $[16,18]$ in the sense that the modular bootstrap of the theory on a torus can lead to the consistent holographic picture. The underlying assumptions in the analysis are that the primary states must have positive norms, while the KacMoody level and the norms of the descendant states are allowed to be negative. It would certainly be interesting to study such "mild nonunitarity" in the supersymmetric case. One may expect the existence of "mild nonunitarity" by allowing a negative Kac-Moody level and only requiring that the superprimary states must have positive norm. The complete analysis on this issue needs to consider the supersymmetric version of CSS boundary conditions.

\section{B. Transformation laws of superprimary fields}

We now consider the transformation laws of the primary superfields. The local operator at position $\left(x^{+}, x^{-}, \theta\right)$ is related to the one at the origin by the transformation,

$$
\Phi(\mathbf{z}) \equiv \Phi\left(x^{+}, x^{-}, \theta\right)=U \Phi(0) U^{-1}, \quad \text { with } \quad U=e^{x^{+} L_{-1}+\theta G_{-\frac{1}{2}}+x^{-} P_{0}} .
$$

Next, we would like to find the explicit form of the commutator $\left[L_{n}, \Phi(\mathbf{z})\right](n \geq 0)$ for a primary field $\Phi(\mathbf{z})$. First, we have

$$
\left[L_{n}, \Phi(\mathbf{z})\right]=U\left[U^{-1} L_{n} U, \Phi(0)\right] U^{-1} .
$$

Using the Baker-Campbell-Hausdorff $(\mathrm{BCH})$ formula, we get

$$
\begin{aligned}
U^{-1} L_{n} U & =\sum_{k=0}^{n+1} \frac{(n+1) !}{k !(n+1-k) !}\left[\left(x^{+}\right)^{k} L_{n-k}+\frac{k}{2}\left(x^{+}\right)^{k-1} \theta G_{n+\frac{1}{2}-k}\right], \\
U^{-1} P_{m} U & =\sum_{k=0}^{m} \frac{m !}{k !(m-k) !}\left[\left(x^{+}\right)^{k} P_{m-k}+\left(x^{+}\right)^{k-1} \theta S_{m+\frac{1}{2}-k}\right], \\
U^{-1} G_{r} U & =\sum_{k=0}^{r+\frac{1}{2}} \frac{\left(r+\frac{1}{2}\right) !}{k !\left(r+\frac{1}{2}-k\right) !}\left(x^{+}\right)^{k} G_{n-k}-\sum_{k=0}^{r+\frac{3}{2}} \frac{\left(r+\frac{1}{2}\right) !}{k !\left(r+\frac{3}{2}-k\right) !}\left[2 k\left(x^{+}\right)^{k-1} \theta L_{r+\frac{1}{2}-k}\right], \\
U^{-1} S_{s} U & =\sum_{k=0}^{s-\frac{1}{2}} \frac{\left(s-\frac{1}{2}\right) !}{k !\left(s-\frac{1}{2}-k\right) !}\left(x^{+}\right)^{k} S_{s-k}-2 \sum_{k=0}^{s+\frac{1}{2}} \frac{\left(s-\frac{1}{2}\right) !}{k !\left(s+\frac{1}{2}-k\right) !} \theta\left(x^{+}\right)^{k-1} P_{s+\frac{1}{2}-k} .
\end{aligned}
$$

Then, we obtain

$$
\begin{aligned}
& {\left[L_{n}, \Phi(\mathbf{z})\right]=U\left[\left(x^{+}\right)^{n+1} L_{-1}+\frac{n+1}{2}\left(x^{+}\right)^{n} \theta G_{-\frac{1}{2}}+(n+1)\left(x^{+}\right)^{n} L_{0}, \Phi(0)\right] U^{-1}, \quad n \geq-1,} \\
& {\left[P_{m}, \Phi(\mathbf{z})\right]=U\left[\left(x^{+}\right)^{m} P_{0}, \Phi(0)\right] U^{-1}, \quad m \geq 0,} \\
& {\left[G_{r}, \Phi(\mathbf{z})\right]=U\left[\left(x^{+}\right)^{r+\frac{1}{2}}\left(G_{-\frac{1}{2}}-2 \theta L_{-1}\right)-2\left(r+\frac{1}{2}\right)\left(x^{+}\right)^{r-\frac{1}{2}} \theta L_{0}, \Phi(0)\right] U^{-1}, \quad r \geq-\frac{1}{2},} \\
& {\left[S_{s}, \Phi(\mathbf{z})\right]=\left(x^{+}\right)^{s-\frac{1}{2}} U\left[-2 \theta P_{0}, \Phi(0)\right] U^{-1}, \quad s>0 .}
\end{aligned}
$$

In particular, we have

$$
\begin{aligned}
& U L_{-1} U^{-1}=L_{-1}, \quad U P_{0} U^{-1}=P_{0}, \\
& U G_{-\frac{1}{2}} U^{-1}=G_{-\frac{1}{2}}+2 \theta L_{-1}, \quad U S_{\frac{1}{2}} U^{-1}=S_{\frac{1}{2}}+2 \theta P_{0} .
\end{aligned}
$$

Using the above relations, we finally obtain

$$
\begin{gathered}
{\left[L_{n}, \Phi(\mathbf{z})\right]=\left[\left(x^{+}\right)^{n+1} \partial_{+}+\frac{n+1}{2}\left(x^{+}\right)^{n} \theta \partial_{\theta}+(n+1)\left(x^{+}\right)^{n} \Delta\right] \Phi(\mathbf{z}), \quad n \geq-1,} \\
{\left[P_{m}, \Phi(\mathbf{z})\right]=\left(x^{+}\right)^{m} \partial_{-} \Phi(\mathbf{z}), \quad m \geq 0,}
\end{gathered}
$$




$$
\begin{gathered}
{\left[G_{r}, \Phi(\mathbf{z})\right]=\left[\left(x^{+}\right)^{r+\frac{1}{2}}\left(\partial_{\theta}-\theta \partial_{+}\right)-2\left(r+\frac{1}{2}\right)\left(x^{+}\right)^{r-\frac{1}{2}} \theta \Delta\right] \Phi(\mathbf{z}), \quad r \geq-\frac{1}{2}} \\
{\left[S_{s}, \Phi(\mathbf{z})\right]=-2\left(x^{+}\right)^{s-\frac{1}{2}} \theta \partial_{-} \Phi(\mathbf{z}), \quad s>0 .}
\end{gathered}
$$

\section{Ward identities and correlation functions}

The vacuum of the NS sector in $\mathrm{SWCFT}_{2}$ is invariant under the global group $\operatorname{OSP}(1 \mid 2) \times U(1)$, which is generated by $\left\{L_{0}, L_{ \pm 1}, P_{0}, G_{ \pm \frac{1}{2}}\right\}$. Thus, the correlation functions obey the Ward identities coming from the generators of $\operatorname{OSP}(1 \mid 2) \times U(1)$. One can solve the differential equations from the Ward identities to find the correlation functions directly using (4.22)-(4.25).

Consider an $n$-point function of superprimary fields,

$$
\begin{aligned}
G^{(n)}\left(\left\{\mathbf{z}_{i}\right\}\right) \equiv & G^{(n)}\left(\left\{x_{i}^{+}, x_{i}^{-}, \theta_{i}\right\}\right) \\
= & \langle 0| T\left[\Phi_{1}\left(x_{1}^{+}, x_{1}^{-}, \theta_{1}\right) \Phi_{2}\left(x_{2}^{+}, x_{2}^{-}, \theta_{2}\right)\right. \\
& \left.\cdots \Phi_{n}\left(x_{n}^{+}, x_{n}^{-}, \theta_{n}\right)\right]|0\rangle
\end{aligned}
$$

where $T$ stands for time ordering, and $\mathbf{z}_{i} \equiv\left\{x_{i}^{+}, x_{i}^{-}, \theta_{i}\right\}$. Throughout this paper, we will always assume $x_{i}^{+}>x_{j}^{+}$for $i<j$. Since the vacuum state $|0\rangle$ is $\operatorname{OSP}(1 \mid 2) \times U(1)$ invariant, the $n$-point function is invariant under the action of $L_{0}, L_{ \pm 1}, P_{0}, G_{ \pm \frac{1}{2}}$. This leads to the following differential equations corresponding to the generators $L_{-1}, P_{0}, L_{0}, L_{+1}, G_{-1 / 2}, G_{1 / 2}$, respectively,

$0=\left(\sum_{i=1}^{n} \partial_{+i}\right) G^{(n)}$

$0=\left(\partial_{-i}-Q_{i}\right) G^{(n)}, \quad$ with $\quad \sum_{i=1}^{n} Q_{i}=0$,

$0=\left(\sum_{i=1}^{n}\left(x_{i}^{+} \partial_{+i}+\frac{1}{2} \theta_{i} \partial_{\theta_{i}}+\Delta_{i}\right)\right) G^{(n)}$,

$0=\left(\sum_{i=1}^{n}\left(\left(x_{i}^{+}\right)^{2} \partial_{+i}+x_{i}^{+} \theta_{i} \partial_{\theta_{i}}+2 x_{i}^{+} \Delta_{i}\right)\right) G^{(n)}$,

$0=\left(\sum_{i=1}^{n} \partial_{\theta_{i}}-\theta_{i} \partial_{+i}\right) G^{(n)}$,

$0=\left(\sum_{i=1}^{n} x_{i}^{+}\left(\partial_{\theta_{i}}-\theta_{i} \partial_{+i}\right)-2 \theta_{i} \Delta_{i}\right) G^{(n)}$.

The first equation implies that $G^{(n)}$ should be a function of $x_{i j}^{+} \equiv x_{i}^{+}-x_{j}^{+}$. While the fifth equation implies that $G^{(n)}$ should be a function of

$$
s_{i j} \equiv x_{i}^{+}-x_{j}^{+}-\theta_{i} \theta_{j},
$$

and

$$
\theta_{i j} \equiv \theta_{i}-\theta_{j}
$$

For the $x^{-}$part, the second equation implies $G^{(n)}$ should be a function of

$$
r_{i j} \equiv x_{i}^{-} Q_{i}+x_{j}^{-} Q_{j}
$$

Consequently, the correlation function is of the form,

$$
G^{(n)}\left(s_{i j}, r_{i j}, \theta_{i j}\right)
$$

We stress that because of the $\operatorname{OSP}(1 \mid 2) \times U(1)$ symmetry of the vacuum, the correlation functions must have the $\operatorname{OSP}(1 \mid 2) \times U(1)$ structure.

Let us first consider the two-point function $G^{(2)}$, which must be of the form,

$$
G^{(2)}\left(\mathbf{z}_{\mathbf{1}}, \mathbf{z}_{2}\right)=\frac{f_{1}\left(r_{12}\right)}{\left(s_{12}\right)^{\kappa_{1}}}+\frac{f_{2}\left(r_{12}\right) \theta_{12}}{\left(s_{12}\right)^{\kappa_{2}}},
$$

where $\left\{f_{i}\right\}$ are the functions to be determined. The equation from the invariance under $P_{0}$ gives

$$
\frac{\partial f_{i}}{\partial r_{12}}=f_{i}, \quad Q_{1}+Q_{2}=0,
$$

which has the solution,

$$
f_{i}=C_{i} e^{r_{12}}, \quad i=1,2,
$$

where $\left\{C_{i}\right\}$ are constants.

Next consider the differential equation arising from dilations $L_{0}$, we find the conditions,

$$
\begin{aligned}
& -\kappa_{1}+\Delta_{1}+\Delta_{2}=0 \\
& -\kappa_{2}+\frac{1}{2}+\Delta_{1}+\Delta_{2}=0 .
\end{aligned}
$$

Moreover, the special transformation $L_{+1}$ leads to the condition,

$$
\begin{aligned}
0= & C_{1}\left(s_{12}\right)^{\frac{1}{2}}\left[\Delta_{12} x_{12}^{+}\right] \\
& +C_{2}\left[\left(\Delta_{12}+\frac{1}{2}\right) x_{12}^{+} \theta_{1}-\left(\Delta_{12}-\frac{1}{2}\right) x_{12}^{+} \theta_{2}\right]
\end{aligned}
$$


which gives

$$
\Delta_{12}=0, \quad C_{2}=0
$$

As a result, we have the two-point function,

$$
G^{(2)}\left(\mathbf{z}_{1}, \mathbf{z}_{2}\right)=\delta_{\Delta_{1}, \Delta_{2}} \delta_{Q_{1},-Q_{2}} \frac{1}{s_{12}^{2 \Delta_{1}}} e^{x_{12}^{-} Q_{1}},
$$

where we have set the normalization to unit. In the component fields, the nonvanishing two-point functions are

$$
\begin{gathered}
\left\langle\varphi_{1} \varphi_{2}\right\rangle=\delta_{\Delta_{1}, \Delta_{2}} \delta_{Q_{1},-Q_{2}} \frac{1}{\left(x_{12}^{+}\right)^{2 \Delta_{1}}} e^{x_{12}^{-} Q_{1}}, \\
\left\langle\psi_{1} \psi_{2}\right\rangle=\delta_{\Delta_{1}, \Delta_{2}} \delta_{Q_{1},-Q_{2}} \frac{2 \Delta_{1}}{\left(x_{12}^{+}\right)^{2 \Delta_{1}+1}} e^{x_{12}^{-} Q_{1}},
\end{gathered}
$$

where $\varphi_{i}$ and $\psi_{i}$ are the component fields of the superfield $\Phi_{i}$.

It is clear that the two-point functions respect the $O S P(1 \mid 2) \times U(1)$ symmetry. The $O S P(1 \mid 2)$ part is determined by the modes $\left\{L_{0}, L_{ \pm 1}, G_{ \pm \frac{1}{2}}\right\}$, just as the usual $N=$ $(1,0) \mathrm{SCFT}_{2}$, while the $U(1)$ part is totally determined by the zero mode $P_{0}$. Moreover, we note that the two-point functions of the superprimaries in $\mathrm{SWCFT}_{2}$ could be obtained straightforwardly from the bosonic one [6] by replacing the difference of the two bosonic coordinates $x_{12}^{+}$ with its supersymmetric generalization $s_{12}$.

From the structures of two-point function, we know that the higher-point correlation functions of $\mathrm{SWCFT}_{2}$ must also include two parts: one being determined by the modes $\left\{L_{0}, L_{ \pm 1}, G_{ \pm \frac{1}{2}}\right\}$, the other being determined by the zero mode $P_{0}$. We can use the well-known results of $N=1$ superconformal theory to determine the $O S P(1 \mid 2)$ structures of the correlation functions of $\mathrm{SWCFT}_{2}$. For example, the holomorphic three-point function in the NS sector of the $N=1$ superconformal theory is given in [40], and the $U(1)$ part is given by [6]; thus, the three-point function in the NS sector of the SWCFT primaries is

$$
\begin{aligned}
& G^{(3)}\left(\mathbf{z}_{\mathbf{1}}, \mathbf{z}_{\mathbf{2}}, \mathbf{z}_{\mathbf{3}}\right) \\
& =\delta_{Q_{1}+Q_{2}+Q_{3}, 0} \frac{C_{123}+\tilde{C}_{123} \Xi_{123}}{s_{12}^{\Delta_{1}+\Delta_{2}-\Delta_{3}} s_{31}^{\Delta_{3}+\Delta_{1}-\Delta_{2}} s_{23}^{\Delta_{2}+\Delta_{3}-\Delta_{1}}} \\
& \quad \times e^{\frac{1}{3} Q_{12} x_{12}^{-}} e^{\frac{1}{3} Q_{31} x_{31}^{-}} e^{\frac{1}{3} Q_{23} x_{23}^{-}},
\end{aligned}
$$

where we have defined $x_{i j}^{-} \equiv x_{i}^{-}-x_{j}^{-}, Q_{i j} \equiv Q_{i}-Q_{j}$ and $\Delta_{i j k} \equiv \Delta_{i}+\Delta_{j}-\Delta_{k}$. The $C_{123}$ and $\tilde{C}_{123}$ are two structure constants of the three-point function. The quantity $\Xi_{i j k}$ is given by

$$
\Xi_{i j k} \equiv \frac{s_{i j} \theta_{k}+s_{j k} \theta_{i}+s_{k i} \theta_{j}}{\sqrt{s_{i j} s_{j k} s_{k i}}} .
$$

For the $n$-point function $(n>3) \mathrm{m}$, there are $3 n$ coordinates $\left\{x_{i}^{+}, x_{i}^{-}, \theta_{i}\right\}$, five constraints from $\operatorname{OSP}(1 \mid 2)$ invariance, and one constraint from $U(1)$ invariance. The $x_{i}^{-}$ dependence can be totally determined by the $U(1)$ invariance; thus, the $n$-point function is essentially a function of $(2 n-5) \operatorname{OSP}(1 \mid 2)$ invariants, which are given by [37]

$$
\Xi_{i j k}, \quad \Theta_{i j k l} \equiv \frac{s_{i j} s_{k l}}{s_{l i} s_{j k}} .
$$

The general form of $n$-point function can be written as

$$
\begin{aligned}
G^{(n)}\left(\left\{\mathbf{z}_{i}\right\}\right)= & \delta \sum_{i=1}^{n} Q_{i}, 0\left(\prod_{i<j=1}^{n} e^{r_{i j}}\right)\left(\prod_{i<j=1}^{n} s_{i j}^{-\Delta_{i j}}\right) \\
& \times F\left(\Xi_{i j k}, \Theta_{i j k l}\right) .
\end{aligned}
$$

Here, $F\left(\Xi_{i j k}, \Theta_{i j k l}\right)$ is an undetermined function, and $\Delta_{i j}$ are real constants, which satisfy

$$
\sum_{i \neq j} \Delta_{i j}=2 \Delta_{j}, \quad \Delta_{i j}=\Delta_{j i}
$$

\section{CONCLUSION AND DISCUSSION}

In the present work, we studied the supersymmetric extension of the warped conformal field theory. Under the assumption that the dilation operator is diagonalizable and has a discrete, non-negative spectrum, we generalized the Hofman-Strominger theorem to the supersymmetric case. Specifically, we showed that a two-dimensional quantum field theory with two translational symmetries, a chiral scaling symmetry, and a chiral supersymmetry may have enhanced local symmetry. The global symmetry could be enhanced to two kinds of minimal algebra. One consists of one copy of the Virasoro algebra and one copy of the superVirasoro algebra, which leads to the $N=(1,0) \mathrm{SCFT}_{2}$. The other consists of one copy of the super-Virasoro algebra plus a $U(1)$ super-Kac-Moody algebra, which leads to the $N=1$ supersymmetric warped conformal field theory.

We discussed some properties of the $\mathrm{SWCFT}_{2}$, including the representations of the algebra, the space of the states, and the transformations of the superfields. We furthermore calculated the two-point and three-point correlation functions of the $\mathrm{SWCFT}_{2}$ with the help of chiral superspace. The form of the correlation functions can be fixed without involving a specific model. Particularly, the vacuum of NS sector in $\mathrm{SWCFT}_{2}$ is $\operatorname{OSP}(1 \mid 2) \times U(1)$ invariant such that the correlation functions must have the $O S P(1 \mid 2) \times U(1)$ structure, in which the $U(1)$ symmetry determined the dependence on $x^{-}$completely. 
For simplicity, our study in Sec. IV focused on the NS sector of the $\mathrm{SWCFT}_{2}$. The discussion can be extended to the Ramond sector; in which case, the mode expansions of the superpartners $G_{r}, S_{r}$ are interger valued. Now the vacuum is doubly degenerated. One may define the superprimary states in the Ramond sector as in usual SCFT. However, we failed to find a spectral flow connecting the NS sector and the Ramond sector. This suggests that there could be some subtlety in the Ramond sector of $\mathrm{SWCFT}_{2}$. We wish to come back to this issue in the future.

One possible future direction is to generalize the minimal supersymmetry to the extended one. Our construction is based on the chiral superspace $\left(x^{+}, \theta\right)$. It is worthy of generalizing the study to the full superspace, including the Grassmannian partner of the $x^{-}$coordinate. In the minimal $\mathrm{CFT}_{2}$ case, this may lead to the $N=(1,1) \mathrm{SCFT}_{2}$. But it is not clear what is the consequence in the $\mathrm{WCFT}_{2}$ case. The study can be pushed to the case of $N \geq 2$ extended supersymmetry as well. Besides, it is interesting to study the supersymmetrization of the other 2D models with scaling symmetry. The supersymmetric GCA has been studied in [41], but for more general anisotropic Galilean field theory [5], its supersymmetric version has not been worked out.

It would be interesting to study the other properties of $\mathrm{SWCFT}_{2}$ : the modular properties of the torus partition function, the warped conformal bootstrap $[6,16]$, the entanglement entropy, etc. It is also interesting to construct explicitly simple examples realizing the $\mathrm{SWCFT}_{2}$. This may help us to understand the theory better.

It could be expected that for the holographic $\mathrm{SWCFT}_{2}$, it is dual to a supersymmetric $\mathrm{AdS}_{3}$ gravity under appropriate asymptotic boundary conditions. It would be nice to find the explicit boundary conditions and see how they break half of the supersymmetries.

\section{ACKNOWLEDGMENTS}

The work was in part supported by NSFC Grants No. 11275010, No. 11335012, No. 11325522, and No. 11735001.

\section{APPENDIX: CONSERVED CHARGES IN THE SUPERSPACE}

We start from the global symmetries of the theory. It is generated by the left-moving translation $H$, the dilation $D$, the right-moving translation $\bar{P}$, and the supersymmetries $Q_{+}$and $Q_{-}$. By assumption, these charges annihilate the vacuum. Their nonvanishing (anti)commutation relations are

$$
i\left\{Q_{+}, Q_{+}\right\}=2 H, \quad i\left\{Q_{+}, Q_{-}\right\}=2 \bar{P}, \quad i\left\{Q_{-}, Q_{-}\right\}=0,
$$

$$
i[D, H]=H, \quad i[D, \bar{P}]=0,
$$

$$
i\left[D, Q_{+}\right]=\frac{1}{2} Q_{+}, \quad i\left[D, Q_{-}\right]=-\frac{1}{2} Q_{-}
$$

The superspace is a coset space $G / I$, where $G$ is the whole symmetry group and $I$ is the dilation symmetry. A group element in $G$ may be written in the form,

$$
g_{0}=e^{i\left(\delta H+\epsilon^{+} Q_{+}+\bar{\delta} \bar{P}+\epsilon^{-} Q_{-}\right)} e^{i \lambda D},
$$

where $\delta, \bar{\delta}, \epsilon^{+}, \epsilon^{-}$, and $\lambda$ are some infinitesimal constants. The coset element can be written as

$$
g_{1}=e^{i\left(x^{+} H+\theta^{+} Q_{+}+x^{-} \bar{P}+\theta^{-} Q_{-}\right)} .
$$

The transformations on the superspace are the natural action of the group $G$ on the coset space,

$$
g_{0} g_{1}=e^{i\left(x^{+} H+\theta^{+\prime} Q_{+}+x^{-\prime} \bar{P}+\theta^{-\prime} Q_{-}\right)} e^{i \lambda^{\prime} D},
$$

from which we read the induced transformations in the superspace,

$$
\begin{gathered}
x^{+\prime}=x^{+}+\delta+\lambda x^{+}-\epsilon^{+} \theta^{+}, \\
\theta^{+\prime}=\theta^{+}+\epsilon^{+}+\frac{\lambda}{2} \theta^{+}, \\
\theta^{-\prime}=\theta^{-}+\epsilon^{-}-\frac{\lambda}{2} \theta^{-}, \\
x^{-\prime}=x^{-}+\bar{\delta}-\epsilon^{-} \theta^{+}-\epsilon^{+} \theta^{-} .
\end{gathered}
$$

Then we can obtain the differential representations of the global charges,

$$
\begin{aligned}
H & =-i \partial_{+}, \quad \bar{P}=-i \partial_{-}, \\
Q_{+} & =-i\left(\partial_{\theta^{+}}-\theta^{+} \partial_{+}-\theta^{-} \partial_{-}\right), \\
Q_{-} & =-i\left(\partial_{\theta^{-}}-\theta^{+} \partial_{-}\right), \\
D & =-i\left(x^{+} \partial_{+}+\frac{\theta^{+}}{2} \partial_{\theta^{+}}-\frac{\theta^{-}}{2} \partial_{\theta^{-}}\right) .
\end{aligned}
$$

For each of the charges $H, D, \bar{P}, Q^{+}$, and $Q^{-}$, there is a conserved Noether current. In particular, with the supersymmetries, there exist corresponding supercurrents. In general, the supercurrents may have the form,

$$
\begin{aligned}
\mathbb{O}_{+}\left(x^{+}, x^{-}, \theta^{+}, \theta^{-}\right)= & a_{1} o_{+1}+a_{2} \theta^{+} o_{+2}+a_{3} \theta^{-} o_{+3} \\
& +a_{4} \theta^{+} \theta^{-} o_{+4}, \\
\mathbb{O}_{-}\left(x^{+}, x^{-}, \theta^{+}, \theta^{-}\right)= & a_{1} o_{-1}+a_{2} \theta^{+} o_{-2}+a_{3} \theta^{-} o_{-3} \\
& +a_{4} \theta^{+} \theta^{-} o_{-4},
\end{aligned}
$$

where $a_{i}(i=1,2,3,4)$ are constant numbers. The charges associated to the components of supercurrents can be read by 


$$
O_{i}=-\frac{1}{2 \pi} \int d x^{+} o_{+i}+\frac{1}{2 \pi} \int d x^{-} o_{-i}
$$

The supersymmetric transformations of the supercurrents are

$$
\begin{aligned}
& i\left[\epsilon_{1} Q_{+}, \mathbb{O}_{+}\left(x^{+}, x^{-}, \theta^{+}, \theta^{-}\right)\right] \\
& =\epsilon_{1}\left(\partial_{\theta^{+}}-\theta^{+} \partial_{+}-\theta^{-} \partial_{-}\right) \\
& \times\left(a_{1} o_{+1}+a_{2} \theta^{+} o_{+2}+a_{3} \theta^{-} o_{+3}+a_{4} \theta^{+} \theta^{-} o_{+4}\right), \\
& =\epsilon_{1}\left(a_{2} o_{+2}-\theta^{+} a_{1} \partial_{+} o_{+1}+\theta^{-}\left(a_{4} o_{+4}-a_{1} \partial_{+} o_{+1}\right)\right. \\
& \left.+\theta^{+} \theta^{-}\left(a_{2} \partial_{-} o_{+2}-a_{3} \partial_{+} o_{+3}\right)\right) \text {, } \\
& i\left[\epsilon_{2} Q_{-}, \mathbb{O}_{+}\left(x^{+}, x^{-}, \theta^{+}, \theta^{-}\right)\right] \\
& =\epsilon_{2}\left(\partial_{\theta^{-}}-\theta^{+} \partial_{-}\right) \\
& \times\left(a_{1} o_{+1}+a_{2} \theta^{+} o_{+2}+a_{3} \theta^{-} o_{+3}+a_{4} \theta^{+} \theta^{-} o_{+4}\right), \\
& =\epsilon_{2}\left(a_{3} o_{+3}-\theta^{+}\left(a_{4} o_{+4}+a_{1} \partial_{-} o_{+1}\right)-a_{3} \theta^{+} \theta^{-} \partial_{+} o_{+3}\right) \text {, }
\end{aligned}
$$

where $\epsilon_{i}(i=1,2)$ are the Grassmannian constants. Then, we have

$$
\begin{aligned}
& i\left[\epsilon_{1} Q_{+}, a_{1} o_{+1}\right]=\epsilon_{1} a_{2} o_{+2}, \\
& i\left[\epsilon_{1} Q_{+}, a_{2} o_{+2}\right]=-\epsilon_{1} a_{1} \partial_{+} o_{+1}, \\
& i\left[\epsilon_{1} Q_{+}, a_{3} o_{+3}\right]=\epsilon_{1}\left(a_{4} o_{+4}-a_{1} \partial_{+} o_{+1}\right), \\
& i\left[\epsilon_{1} Q_{+}, a_{4} o_{+4}\right]=\epsilon_{1}\left(a_{2} \partial_{-} o_{+2}-a_{3} \partial_{+} o_{+3}\right),
\end{aligned}
$$

and

$$
\begin{aligned}
& i\left[\epsilon_{2} Q_{-}, a_{1} o_{+1}\right]=\epsilon_{2} a_{3} o_{+3}, \\
& i\left[\epsilon_{2} Q_{-}, a_{2} o_{+2}\right]=-\epsilon_{2}\left(a_{4} o_{+4}+a_{1} \partial_{-} o_{+1}\right), \\
& i\left[\epsilon_{2} Q_{-}, a_{3} o_{+3}\right]=0, \\
& i\left[\epsilon_{2} Q_{-}, a_{4} o_{+4}\right]=-\epsilon_{2} a_{3} \partial_{+} o_{+3},
\end{aligned}
$$

and similarly, for $\mathbb{O}_{-}\left(x^{+}, x^{-}, \theta^{+}, \theta^{-}\right)$. After the integration, we get the transformations of the charges associated to the components of supercurrents,

$i\left[\epsilon_{1} Q_{+}, a_{1} O_{1}\right]=\epsilon_{1} a_{2} O_{2}, \quad i\left[\epsilon_{1} Q_{+}, a_{2} O_{2}\right]=0$,

$i\left[\epsilon_{1} Q_{+}, a_{3} O_{3}\right]=\epsilon_{1} a_{4} O_{4}, \quad i\left[\epsilon_{1} Q_{+}, a_{4} O_{4}\right]=0$,

$i\left[\epsilon_{2} Q_{-}, a_{1} O_{1}\right]=\epsilon_{2} a_{3} O_{3}, \quad i\left[\epsilon_{2} Q_{-}, a_{2} O_{2}\right]=-\epsilon_{2} a_{4} O_{4}$,

$i\left[\epsilon_{2} Q_{-}, a_{3} O_{3}\right]=0, \quad i\left[\epsilon_{2} Q_{-}, a_{4} O_{4}\right]=0$.

In the following, we will donate the currents associated to the charges $H, \bar{P}, Q_{+}$, and $Q_{-}$by $h_{ \pm}, p_{ \pm}, q_{+ \pm}$, and $q_{- \pm}$, respectively. As it is not clear at this moment how these currents are related to each other by the supersymmetries, we first assume each of them belongs to a supercurrent donated by $\mathbb{U}_{ \pm}, \overline{\mathbb{P}}_{ \pm}, \mathbb{Q}_{+ \pm}$, and $\mathbb{Q}_{- \pm}$, then we will find out the relationship between the currents by their transformations under the supersymmetries. For $\mathbb{Q}_{+ \pm}$, in order to be consistent with (A18) and (A19), it must satisfy

$\begin{array}{rlrl}i\left[\epsilon_{1} Q_{+}, a_{1} Q_{+1}\right] & =\epsilon_{1} a_{2} Q_{+2}, & & i\left[\epsilon_{1} Q_{+}, a_{2} Q_{+2}\right]=0, \\ i\left[\epsilon_{1} Q_{+}, a_{3} Q_{+3}\right]=\epsilon_{1} a_{4} Q_{+4}, & i\left[\epsilon_{1} Q_{+}, a_{4} Q_{+4}\right]=0,\end{array}$

and

$i\left[\epsilon_{2} Q_{-}, a_{1} Q_{+1}\right]=\epsilon_{2} a_{3} Q_{+3}, \quad i\left[\epsilon_{2} Q_{-}, a_{2} Q_{+2}\right]=-a_{4} \epsilon_{2} Q_{+4}$,

$i\left[\epsilon_{2} Q_{-}, a_{3} Q_{+3}\right]=0, \quad i\left[\epsilon_{2} Q_{-}, a_{4} Q_{+4}\right]=0$.

We have similar relations for $\mathbb{Z}_{ \pm}, \overline{\mathbb{P}}_{ \pm}$, and $\mathbb{Q}_{- \pm}$. From these relations, we find that the form of $\mathbb{Q}_{+}$can only be

$$
\mathbb{Q}_{+ \pm}=a_{1} q_{+ \pm}+a_{2} \theta^{+} h_{ \pm}+a_{3} \theta^{-} p_{ \pm},
$$

and the form of $\mathbb{Q}_{-}$must be

$$
\mathbb{Q}_{- \pm}=a_{1} q_{- \pm}+a_{2} \theta^{+} p_{ \pm},
$$

with all nonzero coefficients $a_{i}$, for $i=1,2,3$. We see that the $\bar{P}$ belongs to two different supermultiplets. On the other hand, the fact that the operator $Q_{-}$is nilpotent indicates that we may consider a smaller superspace. In fact, we can regard $Q_{-}$as the superpartner of $\bar{P}$ and consider only one global supercharge $Q_{+}$in the theory. It turns out that the smaller superspace $\left\{x^{+}, x^{-}, \theta^{+}\right\}$is enough to describe our theories consistently.

The superspace $\left\{x^{+}, x^{-}, \theta^{+}\right\}$is the coset space $G / \tilde{I}$, where $G$ is the whole symmetry group and $\tilde{I}$ consists of the dilation symmetry and $Q_{-}$. In this smaller superspace, we have

$$
a_{3}=a_{4}=0, \quad a_{2}=2 a_{1},
$$

and

$$
\mathbb{Q}_{ \pm}=\mathbb{Q}_{+ \pm}, \quad \overline{\mathbb{P}}_{ \pm}=\mathbb{Q}_{- \pm}
$$

We may choose $a_{1}=1$ and find that

$$
\begin{aligned}
& \mathbb{W}_{ \pm}=\mathbb{Q}_{+ \pm}=h_{0 \pm}\left(x^{+}, x^{-}\right)+2 \theta^{+} h_{1 \pm}\left(x^{+}, x^{-}\right), \\
& \overline{\mathbb{P}}_{ \pm}=\mathbb{Q}_{- \pm}=p_{0 \pm}\left(x^{+}, x^{-}\right)+2 \theta^{+} p_{1 \pm}\left(x^{+}, x^{-}\right) .
\end{aligned}
$$


[1] J. Polchinski, Scale and conformal invariance in quantum field theory, Nucl. Phys. B303, 226 (1988).

[2] D. M. Hofman and A. Strominger, Chiral Scale and Conformal Invariance in 2D Quantum Field Theory, Phys. Rev. Lett. 107, 161601 (2011).

[3] A. A. Belavin, A. M. Polyakov, and A. B. Zamolodchikov, Infinite conformal symmetry in two-dimensional quantum field theory, Nucl. Phys. B241, 333 (1984).

[4] S. Detournay, T. Hartman, and D. M. Hofman, Warped conformal field theory, Phys. Rev. D 86, 124018 (2012).

[5] B. Chen, P.X. Hao, and Z.F. Yu, 2d Galilean field theories with anisotropic scaling, Phys. Rev. D 101, 066029 (2020).

[6] W. Song and J. Xu, Correlation functions of warped CFT, J. High Energy Phys. 04 (2018) 067.

[7] G. Compère, W. Song, and A. Strominger, Chiral Liouville gravity, J. High Energy Phys. 05 (2013) 154.

[8] A. Castro, D. M. Hofman, and G. Sárosi, Warped Weyl fermion partition functions, J. High Energy Phys. 11 (2015) 129.

[9] D. M. Hofman and B. Rollier, Warped conformal field theory as lower spin gravity, Nucl. Phys. B897, 1 (2015).

[10] K. Jensen, Locality and anomalies in warped conformal field theory, J. High Energy Phys. 12 (2017) 111.

[11] P. Chaturvedi, Y. Gu, W. Song, and B. Yu, A note on the complex SYK model and warped CFTs, J. High Energy Phys. 12 (2018) 101.

[12] R. A. Davison, W. Fu, A. Georges, Y. Gu, K. Jensen, and S. Sachdev, Thermoelectric transport in disordered metals without quasiparticles: The Sachdev-Ye-Kitaev models and holography, Phys. Rev. B 95, 155131 (2017).

[13] A. Castro, D. M. Hofman, and N. Iqbal, Entanglement entropy in warped conformal field theories, J. High Energy Phys. 02 (2016) 033.

[14] W. Song, Q. Wen, and J. Xu, Modifications to holographic entanglement entropy in warped CFT, J. High Energy Phys. 02 (2017) 067.

[15] T. Azeyanagi, S. Detournay, and M. Riegler, Warped black holes in lower-spin gravity, Phys. Rev. D 99, 026013 (2019).

[16] L. Apolo and W. Song, Bootstrapping holographic warped CFTs or: How I learned to stop worrying and tolerate negative norms, J. High Energy Phys. 07 (2018) 112.

[17] L. Apolo, S. He, W. Song, J. Xu, and J. Zheng, Entanglement and chaos in warped conformal field theories, J. High Energy Phys. 04 (2019) 009.

[18] W. Song and J. Xu, Structure constants from modularity in warped CFT, J. High Energy Phys. 10 (2019) 211.

[19] G. Compère, W. Song, and A. Strominger, New boundary conditions for AdS3, J. High Energy Phys. 05 (2013) 152.

[20] A. Castro, C. Keeler, and P. Szepietowski, Tweaking oneloop determinants in $\mathrm{AdS}_{3}$, J. High Energy Phys. 10 (2017) 070 .
[21] Q. Wen, Towards the generalized gravitational entropy for spacetimes with non-Lorentz invariant duals, J. High Energy Phys. 01 (2019) 220.

[22] B. Chen, P. X. Hao, and W. Song, Rényi mutual information in holographic warped CFTs, J. High Energy Phys. 10 (2019) 037.

[23] Y.h. Lin and B. Chen, Note on bulk reconstruction in $\mathrm{AdS}_{3}$ /warped CFT 2 , Phys. Rev. D 100, 086018 (2019).

[24] B. Gao and J. Xu, Holographic entanglement entropy in $\mathrm{AdS}_{3} / \mathrm{WCFT}$, arXiv:1912.00562.

[25] G. Compere and S. Detournay, Boundary conditions for spacelike and timelike warped $\mathrm{AdS}_{3}$ spaces in topologically massive gravity, J. High Energy Phys. 08 (2009) 092.

[26] A. Aggarwal, A. Castro, and S. Detournay, Warped symmetries of the Kerr Black Hole, J. High Energy Phys. 01 (2020) 016.

[27] M. Ghodrati, Complexity and emergence of warped $\mathrm{AdS}_{3}$ space-time from chiral Liouville action, J. High Energy Phys. 02 (2020) 052.

[28] S. Detournay, W. Merbis, G. S. Ng, and R. Wutte, Warped flatland, arXiv:2001.00020.

[29] R. Andringa, E. Bergshoeff, S. Panda, and M. de Roo, Newtonian gravity and the Bargmann algebra, Classical Quantum Gravity 28, 105011 (2011).

[30] M. H. Christensen, J. Hartong, N. A. Obers, and B. Rollier, Torsional Newton-Cartan geometry and Lifshitz holography, Phys. Rev. D 89, 061901 (2014).

[31] K. Jensen, On the coupling of Galilean-invariant field theories to curved spacetime, SciPost Phys. 5, 011 (2018).

[32] E. A. Bergshoeff, J. Hartong, and J. Rosseel, Torsional Newton-Cartan geometry and the Schrödinger algebra, Classical Quantum Gravity 32, 135017 (2015).

[33] J. Hartong, E. Kiritsis, and N. A. Obers, Lifshitz space-times for Schrödinger holography, Phys. Lett. B 746, 318 (2015).

[34] J. Hartong, E. Kiritsis, and N. A. Obers, Schrödinger invariance from Lifshitz isometries in holography and field theory, Phys. Rev. D 92, 066003 (2015).

[35] P. Di Vecchia, V. G. Knizhnik, J. L. Petersen, and P. Rossi, A supersymmetric Wess-Zumino Lagrangian in two-dimensions, Nucl. Phys. B253, 701 (1985).

[36] I. N. McArthur, Characters of super Kac-Moody algebras, Nucl. Phys. B340, 148 (1990).

[37] J. Fuchs, Superconformal ward identities and the WZW model, Nucl. Phys. B286, 455 (1987).

[38] E. B. Kiritsis and G. Siopsis, Operator algebra of the $N=1$ super Wess-Zumino model, Phys. Lett. B 184, 353 (1987).

[39] J. Polchinski, String theory. Vol. 2: Superstring theory and beyond, https://doi.org/10.1017/CBO9780511618123.

[40] Z. Qiu, Supersymmetry, Two-dimensional critical phenomena and the tricritical Ising model, Nucl. Phys. B270, 205 (1986).

[41] I. Mandal, Supersymmetric extension of GCA in 2d, J. High Energy Phys. 11 (2010) 018. 